\title{
Apatinib treatment for KIT- and KDR-amplified angiosarcoma: a case report

Lishu Yang ${ }^{1}$ Lizhu Liu', Bo Han ${ }^{1 *}$, Wei Han² and Meng Zhao ${ }^{3}$

\begin{abstract}
Background: Metastatic or relapsed angiosarcoma has a poor prognosis and the efficacy of conventional chemotherapy is often limited. Apatinib, a novel tyrosine kinase inhibitor (TKI) targeting vascular endothelial growth factor receptor-2 (VEGFR2), has been approved for the treatment of advanced gastric cancer.

Case presentation: Herein, we report a patient with advanced angiosarcoma, who received apatinib at a daily dose of 250 to $725 \mathrm{mg}$, resulting in a partial response for three months, which may be related to Kinase Insert Domain Receptor (KDR) gene amplification.

Conclusion: Our experience reported here indicated that apatinib may be a useful therapeutic option for treatment of patients with advanced angiosarcoma.
\end{abstract}

Keywords: Apatinib, Angiosarcoma, KDR, Gene-amplified

\section{Background}

Patients with metastatic or relapsed angiosarcoma initially respond well to cytotoxic chemotherapy, however improvements are often short-lived, with a median time to progression averaging four to five months $[1,2]$. Therefore, there is an urgent need for developing efficacious and safe therapies for the treatment of angiosarcoma. Vascular endothelial growth factor receptors (VEGFRs)-targeted therapy has been suggested as a promising approach to the effective treatment of angiosarcoma [3]. Unfortunately, clinical trials with bevacizumab or sorafenib monotherapies in patients with angiosarcoma have thus far shown limited efficacy, with response rates reported as 9 to $14 \%[4,5]$. Herein, we report a patient with a scalp angiosarcoma who responded to apatinib, a novel VEGFR2 inhibitor. We suggest that apatinib should be considered in the management of advanced angiosarcoma.

\section{Case presentation}

A 67-year-old Chinese man appeared with a growing nodular, vascular tumor (enlarging from $0.5 \times 0.5 \mathrm{~cm}$ to $4 \times 3 \mathrm{~cm}$ in nine months) with pruritus and ulcers in the left parietal

\footnotetext{
*Correspondence: bohan1964@163.com

'Department of Oncology, First Affiliated Hospital of Harbin Medical

University, Harbin 150001, China

Full list of author information is available at the end of the article
}

region of the scalp. The patient presented to the neurosurgery clinic of our hospital in November 2015. A computed tomography (CT) scan of the chest, abdomen, and pelvis showed no evidence of metastatic disease. The patient underwent macroscopic radical resection of the tumor and free skin graft repair. Histopathology analysis revealed diffuse distribution of tumor cells with an invasive growth pattern in the epidermis and dermis. Tumor cells were oval and spindle-shaped and showed obvious cellular atypia. Lumen-like structures and massive tumor necrosis was observed (Fig. 1) as angiosarcoma of corium and subcutaneous with infiltration into the muscles and the soft tissues. Histoimmunnochemistry analysis revealed a typical vascular endothelium CD31 (+) and CD34 (+), and lymphatic endothelium D2-40 (+) with a high Ki-67 index (50\%). A computed tomography (CT) scan of the chest, abdomen, and pelvis showed no evidence of metastatic disease. The patient refused to undergo adjuvant chemotherapy and/or radiotherapy as recommended. One month after surgery, ${ }^{18}$ F-FDG PET/CT scan showed evidence of metastasis to liver and bone, with local recurrence occurred two months later. The patient was hospitalized in May 2016 due to scalp angiosarcoma recurrence at six months post-surgery. The patient did not experience any systemic symptoms during the post-operative period. With respect to the patient's medical history, the patient had a history of hypertension for five years, but denied any family history of malignancy. 


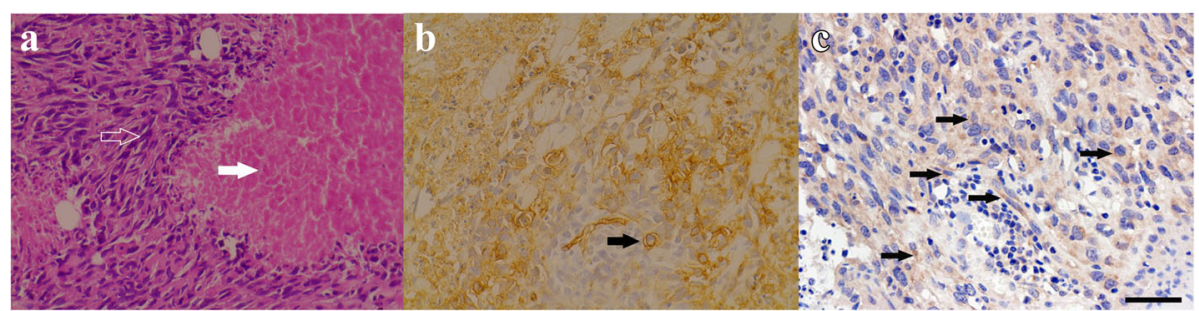

Fig. 1 Hematoxylin and eosin (H\&E) staining of a tumor section. $\mathbf{a} \times 400$; The tumor cells are spindle shaped with obvious heteromorphism(white arrow), and large areas of necrosis(hollow arrow) are visible; $\mathbf{b} \times 400$. CD34 cell membrane positive(black arrow). $\mathbf{c} \times 400$. Immunohistochemistry demonstrates expression of VEGFR2 with color brown (black arrow)

Physical examination showed pink nodules with ulceration in the parietal and frontal regions of the scalp, and nodular lesions anterior to the left ear. A CT scan of chest and abdomen showed multiple solid nodules in the lungs and liver. Radiotherapy was not considered necessary due to widespread recurrent and metastatic diseases. This patient refused chemotherapy.

Considering that KDR gene amplification [2], KDR gene mutation and strong KDR (VEGFR2) expression in angiosarcoma $[6,7]$ have been previously reported, apatinib was given after the patient provided written informed consent. Apatinib was administrated at a dose of $250 \mathrm{mg} /$ day in May 2016, increasing to $675 \mathrm{mg} /$ day after five days. Resected angiosarcoma tissue from the scalp was analyzed for gene mutations, and demonstrated KIT amplification (8 copies), KDR amplification (8 copies) (Fig. 2), RB1 codon 251 arginine mutated to a stop codon, and TP53 codon 195 isoleucine mutated to threonine with a reduced copy number. After three weeks of apatinib treatment, the swelling of scalp and forehead was markedly reduced (Fig. 3) and the CT scan of chest showed significant reduction of solid nodules and transformation of solid nodules into cystic nodules (Fig. 4). Physical examination conducted in July 2016 showed no progressive disease, a closed pneumothorax of the right thoracic cavity was found after 10 weeks of apatinib administration, with suspected side-effects, such as glossodynia, odynophagia, upper abdominal pain, melena, hemoptysis, and delayed healing of the lesion of scalp after tumor regression. Medication was stopped for one week in order to promote the recovery of the left-sided pneumothorax after 11 weeks of administration of apatinib. The patient then received a reduced daily dose of $425 \mathrm{mg}$ apatinib for three weeks. When closure of the pneumothorax of right thoracic happened, the medication had to be interrupted again, and disease progression was observed in scalp, and lungs and liver proven by $\mathrm{CT}$ scan. Further therapy was limited because of his severe comorbidities. The

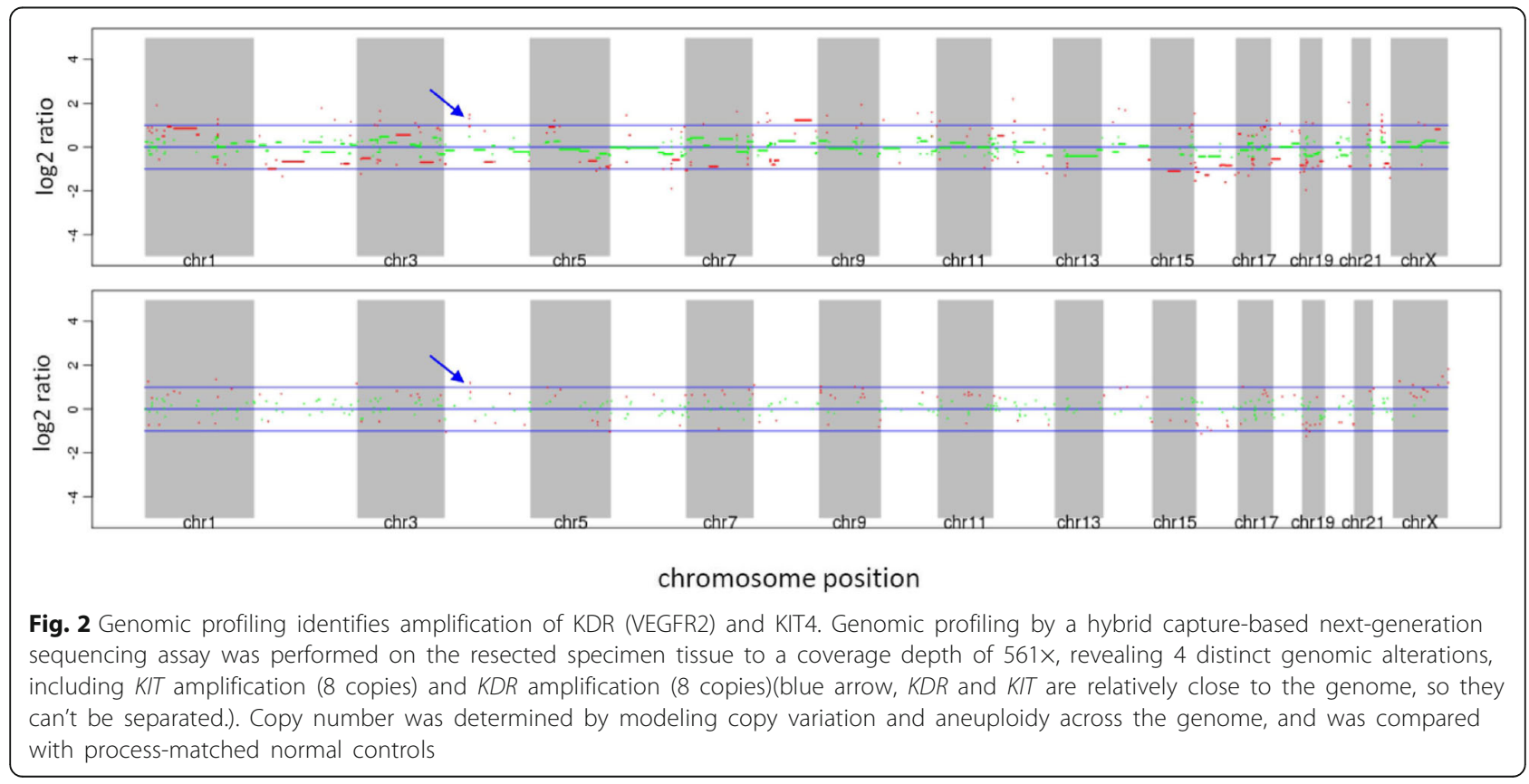



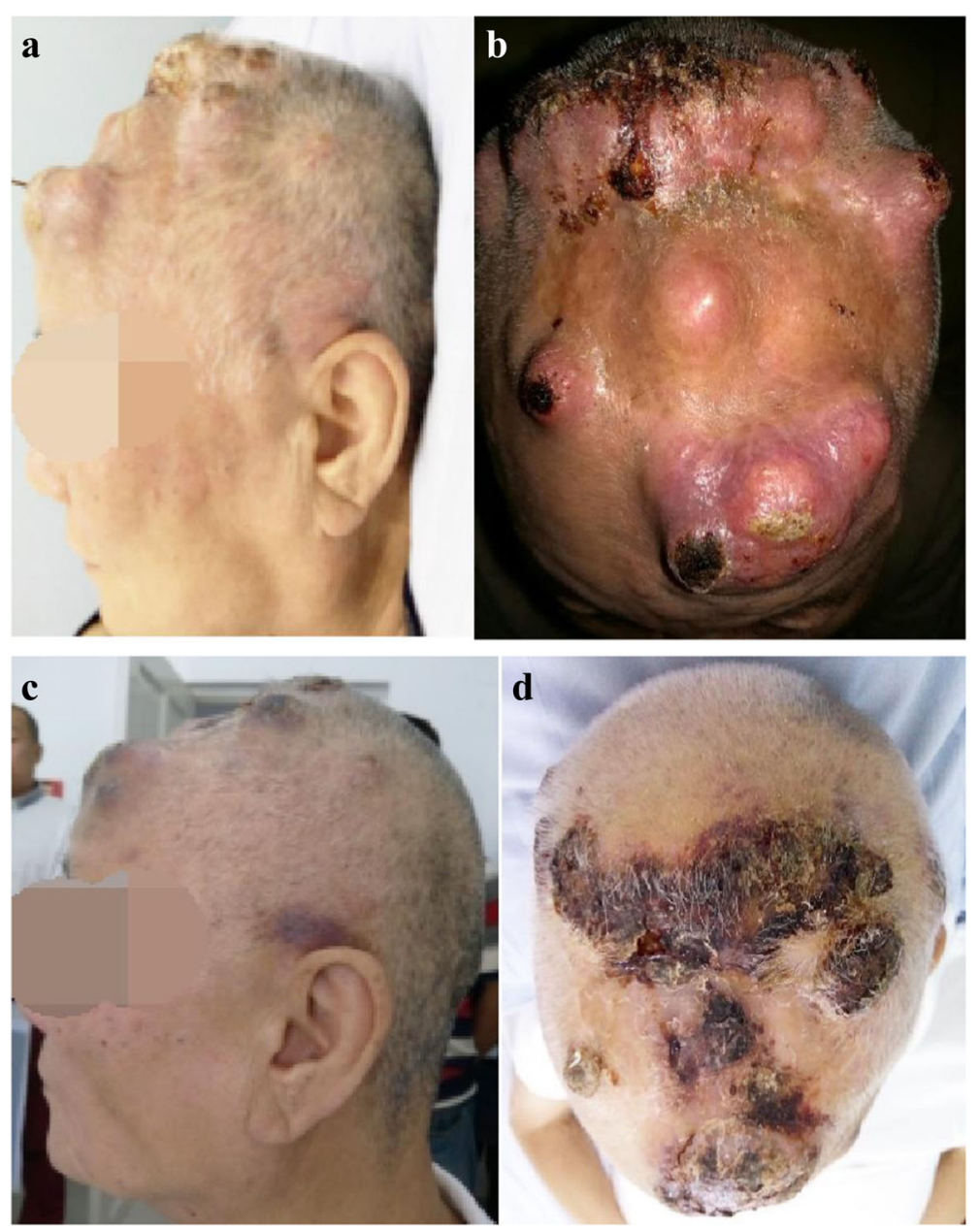

Fig. 3 Extensive cutaneous angiosarcoma of the scalp. ( $(\mathbf{a}$ and $\mathbf{b})$ Before initiation of apatinib. $\mathbf{c}$ After three weeks of treatment with oral apatinib at $675 \mathrm{mg}$ daily (d) After eight weeks of treatment with oral apatinib at $675 \mathrm{mg}$ daily

patient subsequently died after his partial response maintained for three months.

\section{Discussion}

The treatment with apatinib in our patient resulted in a three-month partial remission (PR). The duration of the PR achieved should be considered acceptable. Based on previous reports, the median progression-free survival time (PFS) and response rate in angiosarcomas are with 3.8 months and $14 \%$ for sorafenib [8] and three months and $9 \%$ for bevacizumab, respectively [9].

Angiosarcoma is a malignant neoplasm of vascular-origin derived from endothelial cells [7]. The NCCN Clinical Practice Guidelines in Oncology (NCCN Guidelines) for

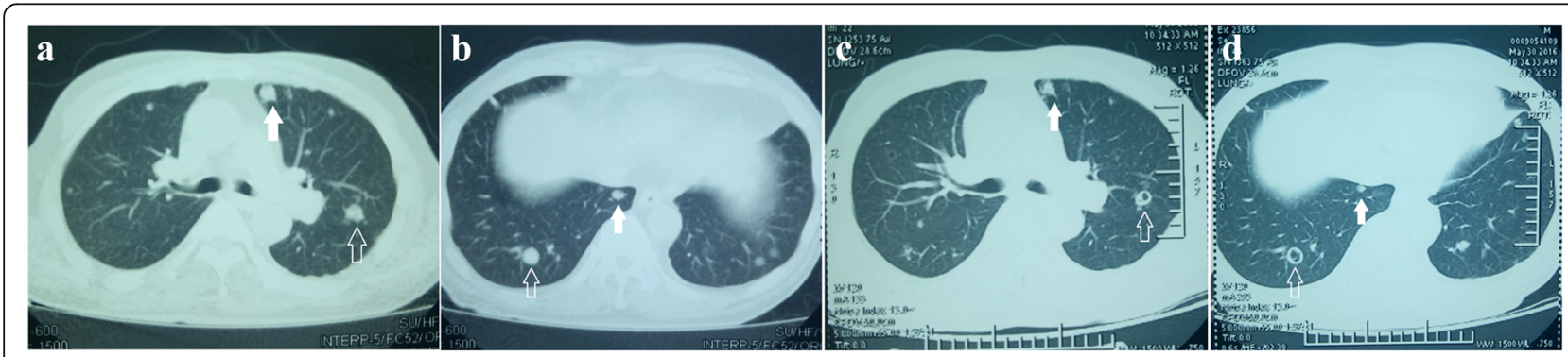

Fig. 4 CT scans showing significant reduction of solid nodules(white arrows) and transform from solid nodules before treatment(hollow arrow) (a and $\mathbf{b}$ ) to cystic nodules(hollow arrow) after 3 weeks of treatment with apatinib (c and $\mathbf{d}$ ) 
Soft Tissue Sarcoma recommends surgery and radiotherapy as the primary mode of treatment for local control of angiosarcoma. Systemic therapy is essential due to high local recurrence rates and distant metastasis risk. Patients with metastatic or relapsed disease often respond well to cytotoxic chemotherapy initially, however the median time to progression is short, often four to five months [10,11].

The use of cytotoxic agents and targeted therapies forms the mainstay of therapy for patients with metastatic or unresectable disease [12] As a novel targeted therapeutic agent, apatinib targets VEGFR signaling, which is frequently implicated in angiosarcoma. Indeed, VEGFR2 regulates angiogenesis [13] and inappropriate activation of VEGFR signaling is critical in the development and progression of angiosarcoma $[2,6,7,14]$. Activating base substitutions in VEGFR2 [7] and VEGFR1-3 overexpression have been demonstrated in patients with angiosarcoma [13, 15]. In order to explore the relationship between KDR gene amplification and VEGFR2 expression, we carried out immunohistochemical detection of VEGFR-2 in the resected tumor tissues, and found a moderate degree of expression (Fig. 1c).

Apatinib is a small-molecule TKI that highly selectively binds to and strongly inhibits VEGFR-2, decreasing VEGF-mediated endothelial cell migration, proliferation, and tumor microvascular formation. It has been shown to be a novel therapeutic option in a variety of tumor types [16]. In a Phase I study, dose escalation of apatinib was conducted from 250 to $1000 \mathrm{mg}$ in patients with solid tumors aged between 18 and 70 years, with the study demonstrating a maximum tolerated dose of $850 \mathrm{mg}$ once daily. The most frequently observed drug-related adverse events were hypertension (69.5\%), proteinuria (47.8\%), and hand-foot syndrome (45.6\%) [1].

Identifying patients with an increased likelihood of response to therapy is a major challenge in the clinic. Identification of genomic alterations may help further sub-classify tumors. Based on our case, there was a possible correlation between responsiveness to apatinib and amplification of $K D R$. Pre-clinical studies have shown that KDR activation variants are more sensitive to sunitinib and sorafenib [7]; indeed, one of the targets of apatinib is KDR [17]. In addition to our findings, there have been some previously published reports with similar findings. Colorectal cancer patients with pulmonary metastases who had a VEGFR2 mutation responded to apatinib treatment [1]. Response to sunitinib has been reported in patients with KIT mutation [3] and in patients with $\mathrm{KDR}$ mutations [18]. The $\mathrm{IC}_{50}$ for apatinib with respect to VEGFR2 is $0.001 \mathrm{M}$, whereas the $\mathrm{IC}_{50}$ for sunitinib is $0.005 \mathrm{M}$ [19] suggesting that apatinib is highly selective. Our case may represent a subset of highly apatinib-responsive angiosarcomas characterized by $K D R$ amplification.
Our patient presented some common adverse events, such as hypertension, proteinuria, and hand-foot syndrome [1], which were not severe and therefore did not require dose reduction or withdrawal of the therapy. However, we also observed other side effects, including glossodynia, odynophagia, upper abdominal pain, melena, hemotpyssis, delayed healing of scalp lesions, and a pneumothorax. These symptoms seriously reduced oral intake and thus worsened the patient's physical condition. The pneumothorax might be the result of tumor regression and indeed, eventually led to withdrawal of the medication. It may also be a feasible choice to maintain a low dose rather than a standard dose if the dose of $250 \mathrm{mg}$ can achieve a significant tumor regression. When drug resistance occurs, the patient might still have an opportunity to receive other targeted drug treatment such as Pazopanib [2], Sunitinib [3], sorafenib [8], and bevacizumab [9]. Therefore, individualized dose scheduling should be considered in order to achieve a balance between tumor regression and tissue repair to expect a longer period of remission.

\section{Conclusion}

The present case demonstrated the potential therapeutic effects of apatinib in patients with metastatic angiosarcoma. Future clinical studies are required to identify a subset of patients with angiosarcomas with well-defined genomic variants sensitive to apatinib. Prospective clinical trials accompanied by comprehensive genomic profiling are needed. Additionally, pharmacogenomics studies will be needed to help define individual dosing and scheduling at the beginning of the therapy and aid in dose adjustment based on patient responses and safety profiles.

\section{Abbreviations \\ KDR: Kinase Insert Domain Receptor; TKI: Tyrosine kinase inhibitor}

\section{Acknowledgements}

The authors thank the patient's relatives for their agreement to publish this report, and thank 3D Medicine Inc. Shanghai, P.R. China for their technical support.

\section{Availability of data and materials \\ The datasets used and/or analyzed during the current study are presented within the manuscript.}

\section{Authors' contributions}

$Y L S, H B, L L Z, H W$, and ZM drafted the manuscript and revised it. YLS, HB, and LLZ made substantial contributions to the conception of the study, design of experiments, and acquisition of data. YLS, HW, and ZM analyzed the data and prepared the figures. All the authors have read the final version of the manuscript and approved the submission.

Ethics approval and consent to participate

This study was approved by the ethical committee of First Affiliated Hospital of Harbin Medical University. Informed written consent was obtained.

\section{Consent for publication}

Written informed consent for publication of their clinical details and clinical images was obtained from the patient. A copy of the consent form is available for review by the Editor of this journal. 


\section{Competing interests}

The authors declare that they have no competing interests.

\section{Publisher's Note}

Springer Nature remains neutral with regard to jurisdictional claims in published maps and institutional affiliations.

\section{Author details}

${ }^{1}$ Department of Oncology, First Affiliated Hospital of Harbin Medical University, Harbin 150001, China. ${ }^{2}$ Department of Pathology, First Affiliated Hospital of Harbin Medical University, Harbin 150001, China. ${ }^{3}$ 3D Medicine Inc. Shanghai, Shanghai201114People's Republic of China.

Received: 21 October 2017 Accepted: 18 May 2018

Published online: 31 May 2018

\section{References}

1. Li J, Zhao X, Chen L, et al. Safety and pharmacokinetics of novel selective vascular endothelial growth factor receptor-2 inhibitor YN968D1 in patients with advanced malignancies. BMC Cancer. 2010;10:529.

2. Ravi V, Sanford EM, Wang WL, et al. Antitumor response of VEGFR2- and VEGFR3-amplified Angiosarcoma to Pazopanib. J Natl Compr Cancer Netw. 2016;14:499-502.

3. Minor DR, Kashani-Sabet M, Garrido M, O'Day SJ, Hamid O, Bastian BC. Sunitinib therapy for melanoma patients with KIT mutations. Clin Cancer Res. 2012;18:1457-63.

4. Ganjoo KN, Villalobos VM, Kamaya A, et al. A multicenter phase II study of pazopanib in patients with advanced gastrointestinal stromal tumors (GIST) following failure of at least imatinib and sunitinib. Ann Oncol. 2014;25:236-40.

5. Joensuu H, Puputti M, Sihto H, Tynninen O, Nupponen NN. Amplification of genes encoding KIT, PDGFRalpha and VEGFR2 receptor tyrosine kinases is frequent in glioblastoma multiforme. J Pathol. 2005;207:224-31.

6. Kristin Kunze, Tilmann Spieker, Ulrike Gamerdinger et al; A Recurrent Activating PLCG1 mutation in cardiac angiosarcomas increases apoptosis resistance and invasiveness of endothelial cells. Published Online First September 24, 2014; DOI: https://doi.org/10.1158/0008-5472.CAN-14-1162.

7. Antonescu CR, Yoshida A, Guo T, et al. KDR activating mutations in human angiosarcomas are sensitive to specific kinase inhibitors. Cancer Res. 2009; 69:7175-9.

8. Maki RG, D'Adamo DR, Keohan ML, et al. Phase II study of sorafenib in patients with metastatic or recurrent sarcomas. J Clin Oncol. 2009;27: 3133-40.

9. Agulnik M, Yarber JL, Okuno SH, et al. An open-label, multicenter, phase ॥ study of bevacizumab for the treatment of angiosarcoma and epithelioid hemangioendotheliomas. Ann Oncol. 2013;24:257-63.

10. Fata F, O'Reilly $E$, Ilson $D$, et al. Paclitaxel in the treatment of patients with angiosarcoma of the scalp or face. Cancer. 1999;86:2034-7.

11. Fury MG, Antonescu CR, Van Zee KJ, Brennan MF, Maki RG. A 14-year retrospective review of angiosarcoma: clinical characteristics, prognostic factors, and treatment outcomes with surgery and chemotherapy. Cancer J. 2005;11:241-7.

12. Margaret von Mehren, R. Lor Randall and Robert S. Benjamin: Systemic Therapy Agents and Regimens with Activity in Soft Tissue Sarcoma Subtypes. NCCN Clinical Practice Guidelines in Oncology; Soft Tissue Sarcoma. Version 2.2016. SARC-F 1-2 OF 6. Accessed 4 Apr 2016.

13. Jussila L, Alitalo K. Vascular growth factors and lymphangiogenesis. Physiol Rev. 2002;82:673-700

14. Park MS, Ravi V, Araujo DM. Inhibiting the VEGF-VEGFR pathway in angiosarcoma, epithelioid hemangioendothelioma, and hemangiopericytoma/solitary fibrous tumor. Curr Opin Oncol. 2010;22:351-5.

15. Cabeza M, Moilanen A. Design of reserve networks and the persistence of biodiversity. Trends Ecol Evol. 2001;16:242-8.

16. Li J, Qin S, Xu J, et al. Randomized, double-blind, placebo-controlled phase iii trial of apatinib in patients with chemotherapy-refractory advanced or metastatic adenocarcinoma of the stomach or gastroesophageal junction. J Clin Oncol. 2016;34:1448-54.
17. Ferrara N, Kerbel RS. Angiogenesis as a therapeutic target. Nature. 2005:438:967-74.

18. Subbiah V, Meric-Bernstam F, Mills GB, et al. Next generation sequencing analysis of platinum refractory advanced germ cell tumor sensitive to Sunitinib (Sutent(R)) a VEGFR2/PDGFRbeta/c-kit/ FLT3/RET/CSF1R inhibitor in a phase II trial. J Hematol Oncol. 2014;7:52.

19. Shu T, Quan HT, Xie CY. YN968D1 is a novel and selective inhibitor of vascular endothelial growth factor receptor-2 tyrosine kinase with potent activity in vitro and in vivo. Cancer Sci. 2011;102:1374-80.

\section{Ready to submit your research? Choose BMC and benefit from:}

- fast, convenient online submission

- thorough peer review by experienced researchers in your field

- rapid publication on acceptance

- support for research data, including large and complex data types

- gold Open Access which fosters wider collaboration and increased citations

- maximum visibility for your research: over $100 \mathrm{M}$ website views per year

At BMC, research is always in progress.

Learn more biomedcentral.com/submissions 